\title{
XMM-Newton observation of the Be/neutron star system RX J0146.9+6121: a soft X-ray excess in a low luminosity accreting pulsar ${ }^{\star}$
}

\author{
N. La Palombara and S. Mereghetti
}

\begin{abstract}
INAF - Istituto di Astrofisica Spaziale e Fisica Cosmica Milano, via Bassini 15, 20133 Milano, Italy e-mail: nicola@iasf-milano.inaf.it
\end{abstract}

Received 28 February 2006 / Accepted 19 April 2006

ABSTRACT

\begin{abstract}
We report on the XMM-Newton observation of the Be/neutron star X-ray binary system RX J0146.9+6121, a long period ( 23 m) pulsar in the NGC 663 open cluster. The X-ray luminosity decreased by a factor of two compared to the last observation carried out in 1998, reaching a level of $\sim 1 \times 10^{34} \mathrm{erg} \mathrm{s}^{-1}$, the lowest ever observed in this source. The spectral analysis reveals the presence of a significant excess at low energies over the main power-law spectral component. The soft excess can be described by a black-body spectrum with a temperature of about $1 \mathrm{keV}$ and an emitting region with a radius of $\sim 140 \mathrm{~m}$. Although the current data do not permit us to ascertain whether the soft excess is pulsed or not, its properties are consistent with emission from the neutron star polar cap. This is the third detection of a soft excess in a low luminosity $\left(\sim 1 \times 10^{34} \mathrm{erg} \mathrm{s}^{-1}\right)$ pulsar, the others being X Per and 3A $0535+262$, suggesting that such a spectral component, observed to date in higher luminosity systems, is a common feature of accreting X-ray pulsars. The results of these three sources indicate that, in low luminosity systems, the soft excess tends to have a higher temperature and a smaller surface area than in the high luminosity ones.
\end{abstract}

Key words. stars: individual: RX J0146.9+6121 - X-rays: binaries

\section{Introduction}

High Mass X-Ray Binaries (HMXRBs) are binary systems consisting of a neutron star (NS) or, less frequently, a black hole, accreting matter from a high mass early type star. Based on the nature of the mass donor star, either a supergiant of $\mathrm{O} / \mathrm{B}$ spectral type or a Be star, HMXRBs can be divided in two subgroups showing different variability properties. HMXRBs with supergiant companions tend to be persistent sources, although some of them display high and low states with X-ray flux varying by large factor ( $\sim 100$ or more). On the other hand, neutron star Be $\mathrm{X}$-ray binaries (BeXRBs) are generally transient sources, due to the long term variability of the equatorial discs surrounding Be stars and/or the orbital eccentricity. For both subgroups, when the compact object is a NS, the X-ray spectra are well described by a rather flat power-law between 0.1 and $10 \mathrm{keV}$ (photon index $\sim 1$ ) followed by a high-energy cutoff.

With the advent of imaging X-ray satellites a large number of BeXRB systems has been discovered in the Small Magellanic Cloud (Haberl \& Sasaki 2000; Israel et al. 2000; Yokogawa et al. 2003; Macomb et al. 2003; Sasaki et al. 2003; Haberl \& Pietsch 2004). The observation of these sources, unaffected by the high interstellar absorption present in the Galactic plane, makes it possible to study in detail their X-ray spectra, extending down to energies of a few hundred $\mathrm{eV}$. This has allowed investigators to discover that most of them have a marked soft excess above the power-law model (Nagase 2002; Haberl \& Pietsch 2005).

^ This work is based on observations obtained with XMM-Newton, an ESA science mission with instruments and contributions directly funded by ESA Member States and NASA.
RX J0146.9+6121 is a BeXRB hosting a neutron star characterized by a rotational period of about $23 \mathrm{~min}$, among the longest observed in X-ray pulsars. The pulsations were discovered with non-imaging instruments on board EXOSAT and initially attributed to a different nearby source (White et al. 1987). Subsequent observations clarified the picture (Mereghetti et al. 1993; Hellier 1994) and led to the optical identification of RX J0146.9+6121 with the B0 IIIe star LS I +61 235 (Coe et al. 1993). This star is a member of the open cluster NGC 663 (Tapia et al. 1991; Fabregat et al. 1996; Pigulski et al. 2001) for which a distance in the range between 2 and $2.5 \mathrm{kpc}$ has been derived (Kharchenko et al. 2005; Pandey et al. 2005). In the following we adopt $d=2.5 \mathrm{kpc}$. Due to its relatively short distance, RX J0146.9+6121 is not much absorbed and is therefore a good target to investigate the properties of the soft X-ray emission in a Galactic source. Here we present the results of a recent observation obtained with the XMM-Newton satellite, providing the most sensitive observation of this source ever obtained below $2 \mathrm{keV}$.

\section{Observations and data reduction}

RX J0146.9+6121 was observed by XMM-Newton between 22:40 UT of 2004 January 14 and 10:20 UT of 2004 January 15 . Since the main target was the NGC 663 open cluster, RX J0146.9+6121 was detected at an off-axis angle of 9.3'. The three EPIC instruments, i.e. the $p n$ camera (Strüder et al. 2001) and the MOS1 and MOS2 cameras (Turner et al. 2001), were active and operated in Full Frame mode. For all of them, the Medium thickness filter was used. 


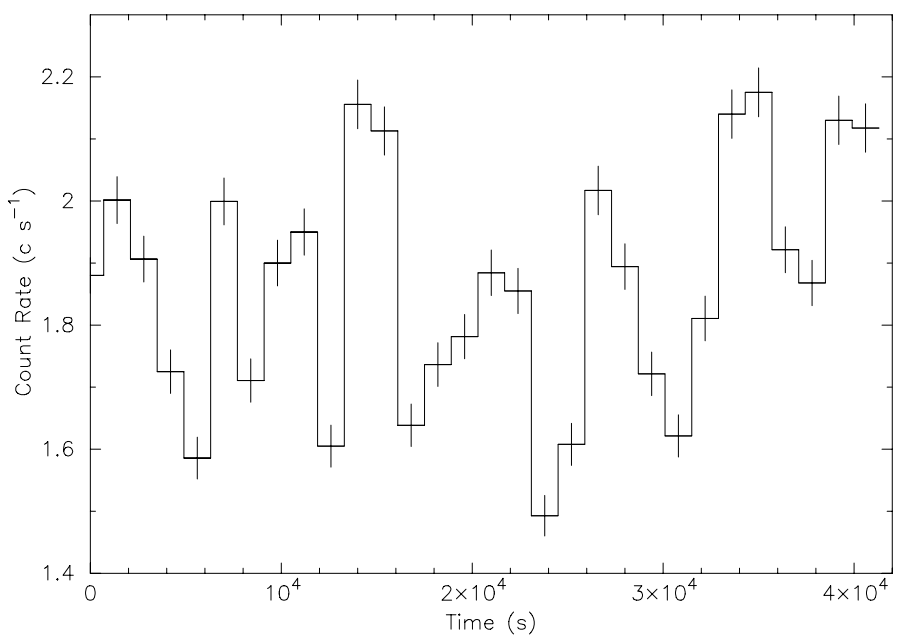

Fig. 1. Background subtracted light curve of RX J0146.9+6121 in the energy range $0.15-10 \mathrm{keV}$, with a time bin of $1.4 \mathrm{ks}$ (i.e. 1 pulse period).

We used version 6.1 of the XMM-Newton Science Analysis System $(S A S)$ to process the event files. After the standard pipeline processing of the data, we looked for possible periods of high instrumental background, due to flares of soft protons with energies less than a few hundred $\mathrm{keV}$. We found that the first $\sim 15 \mathrm{ks}$ of the observation were affected by a soft-proton contamination. However, since RX J0146.9+6121 was detected with a count-rate $\left(\sim 1 \mathrm{cts} \mathrm{s}^{-1}\right)$ much higher than that of the background, the soft-protons during the bad time intervals have a negligible effect on the source spectral and timing analysis (their count rate in the source extraction area is less than $0.01 \mathrm{cts} \mathrm{s}^{-1}$ ). Hence for our analysis we used the data of the whole observation, corresponding to exposure times of 35.4 and $41.2 \mathrm{ks}$ in the $p n$ and $M O S$ cameras, respectively.

\section{Timing analysis}

During our observation some flux changes on an $\sim$ hour timescale were present, as shown by the background subtracted light curve plotted in Fig. 1. This is based on the data in the $0.15-10 \mathrm{keV}$ range obtained from the three EPIC cameras. A bin size of $1.4 \mathrm{ks}$, corresponding to one spin period, has been chosen to avoid the effects due to the periodic pulsations. Variations up to $\sim 20 \%$ around the average level of $\sim 1.9 \mathrm{cts} \mathrm{s}^{-1}$ are evident.

To obtain a measure of the pulse period, we converted the times of arrival to the solar system barycenter and performed a folding analysis using the source events of three cameras. By fitting the $\chi^{2}$ versus trial period curve with the appropriate sinc function as described in Leahy (1987), we derived a period of $1396.14 \pm 0.25$ s. In Fig. 2 we show the folded light curves in four energy intervals $(0.3-2,2-4.5,4.5-7$ and $7-10 \mathrm{keV})$ chosen to allow a direct comparison with the light curves previously derived with RossiXTE data (Mereghetti et al. 2000). The pulse profile, characterized by a broad peak, is clearly energydependent, with the maximum shifting from phase $\sim 0.4-0.5$ at $E<2 \mathrm{keV}$ to phase $\sim 0.6$ at $E>7 \mathrm{keV}$.

The hardness ratio (HR) between the light curves above and below $2 \mathrm{keV}$, reported in Fig. 3, is characterized by a slow and regular increase from its minimum to its maximum value, followed by a rather irregular decrease. This plot, where we have divided the pulse period in ten phase intervals, shows that there is not a simple correlation between the hardness and the total count rate: we observe the same HR value at completely

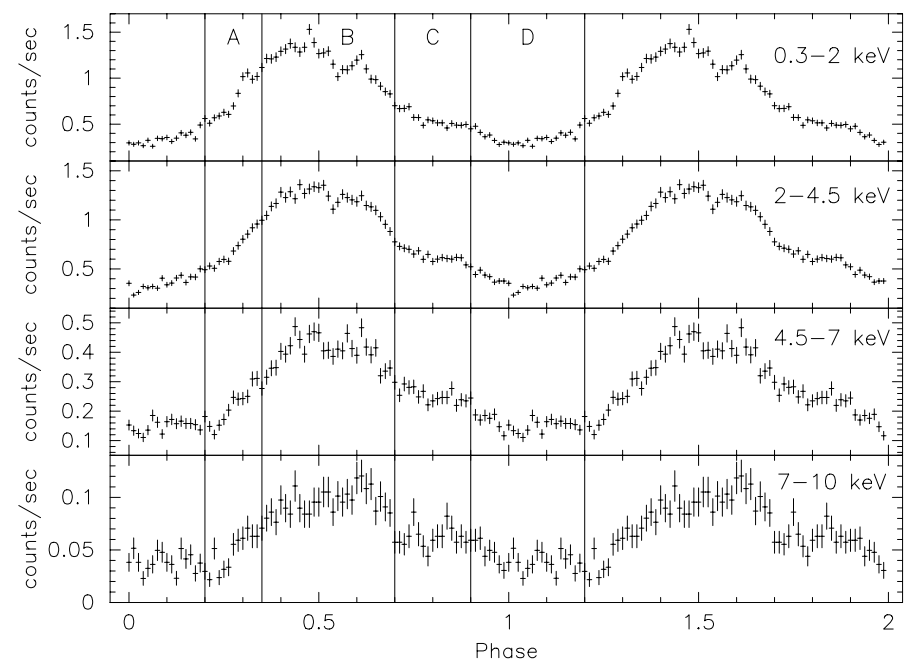

Fig. 2. Background subtracted light curves of RX J0146.9+6121 in the energy ranges $0.3-2,2-4.5,4.5-7$ and $7-10 \mathrm{keV}$, folded at the best-fit period of $1396.14 \mathrm{~s}$. The vertical lines indicate the phase intervals used for the spectral analysis.

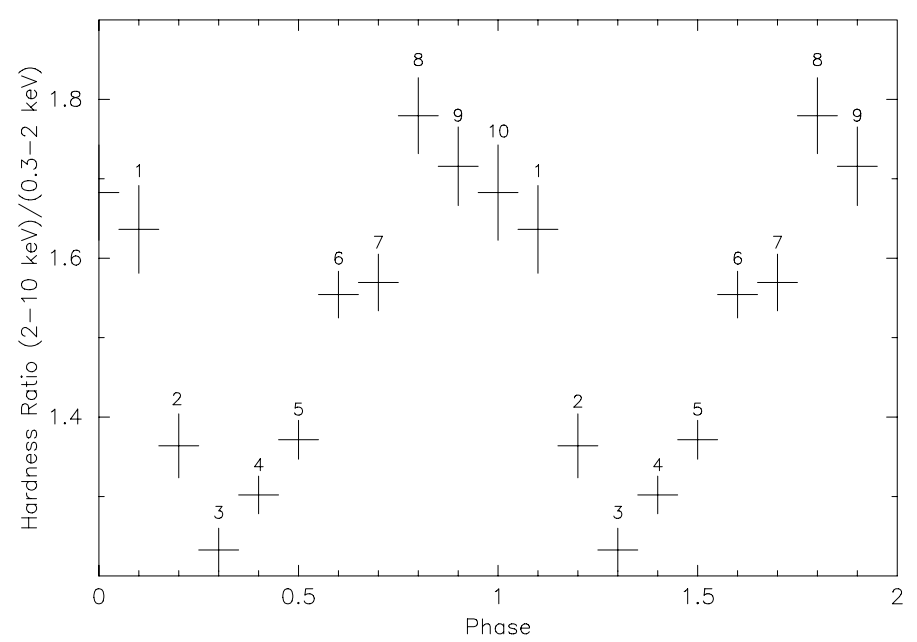

Fig. 3. Hardness ratio of the two light curves of RX J0146.9+6121 in the energy ranges $0.3-2$ and $2-10 \mathrm{keV}$, as a function of the pulse phase. The HR values are obtained by folding the light curves at the best-fit period of $1396.14 \mathrm{~s}$. The pulse period is divided in 10 phase bins, which are identified by progressive numbers.

different count rate levels, but also very different HR values for the same count rate. This indicates that the spectral hardness of RX J0146.9+6121 does not depend in a simple way on its flux level.

\section{Spectral analysis}

For the source spectra we used an extraction radius of $30^{\prime \prime}$ around the source position in the case of the MOS 2 and pn cameras; for the MOS1 camera the extraction radius was reduced to $15^{\prime \prime}$, since the source was imaged close to a CCD gap. We checked with the SAS task epatplot that no event pile-up affected our data, then we accumulated all the events with pattern range 0-4 (i.e. mono- and bi-pixel events) and 0-12 (i.e. from 1 to 4 pixel events) for the $p n$ and the two MOS cameras, respectively. The background spectra were accumulated on large circular areas with no sources and radius of $210^{\prime \prime}$ and $120^{\prime \prime}$ for the MOS1 and the MOS 2 camera, respectively. For the $p n$ camera 


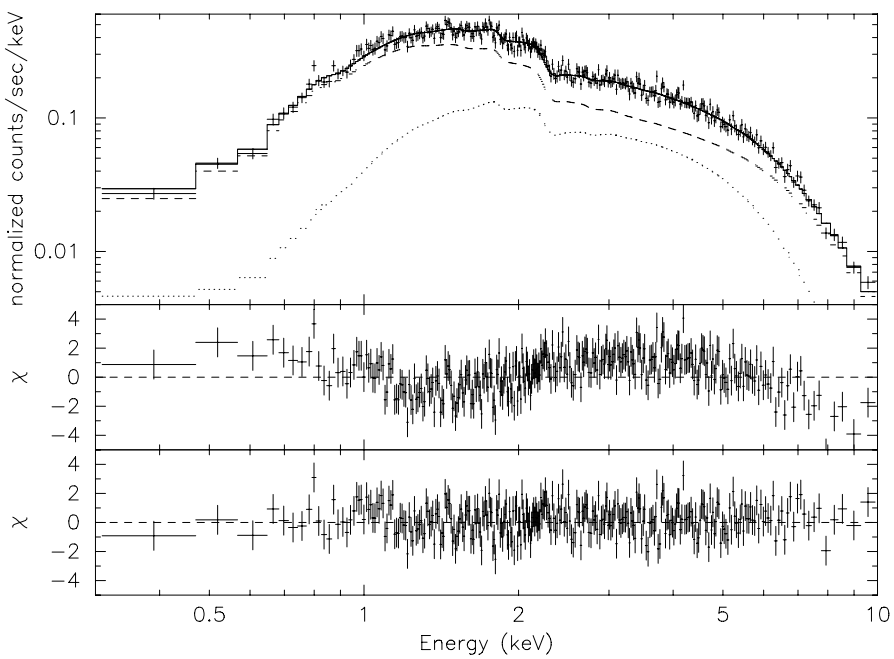

Fig. 4. Top panel: pn spectrum of RX J0146.9+6121 with the best-fit power-law (dashed line) plus black-body (dotted line) model. Middle panel: residuals (in units of $\sigma$ ) between data and model in the case of the single power-law. Bottom panel: residuals in the case of the powerlaw plus black-body.

the background spectrum was extracted from a circular region of the same radius and at the same CCD rows of the source region. We generated ad hoc response matrices and ancillary files using the SAS tasks rmfgen and arfgen. All spectra were rebinned with a minimum of 30 counts per bin and fitted in the energy range 0.3-10 keV using XSPEC 11.3.2.

After checking that separate fits of the three spectra gave consistent results, we fitted the spectra from the three cameras simultaneously in order to increase the count statistics and to reduce the uncertainties. The fit with an absorbed power-law yielded $N_{\mathrm{H}}=(6.24 \pm 0.14) \times 10^{21} \mathrm{~cm}^{-2}$ and photon index $\Gamma=$ $1.36 \pm 0.02$, but with large residuals and $\chi_{v}^{2} /$ d.o.f. $=1.283 / 1480^{1}$. The addition of a blackbody component improved the fit quality significantly (Fig. 4): we obtained $N_{\mathrm{H}}=\left(5.09_{-0.23}^{+0.24}\right) \times 10^{21} \mathrm{~cm}^{-2}$, $\Gamma=1.34_{-0.06}^{+0.05}$ and $k T_{\mathrm{BB}}=1.11_{-0.06}^{+0.07} \mathrm{keV}$, with $\chi_{v}^{2} /$ d.o.f. $=$ $1.036 / 1478$. The emission surface of the thermal component has a radius $R_{\mathrm{BB}}=140_{-14}^{+17} \mathrm{~m}$ (for $d=2.5 \mathrm{kpc}$ ). The unabsorbed flux in the energy range $0.3-10 \mathrm{keV}$ is $f_{\mathrm{X}} \sim 2 \times 10^{-11} \mathrm{erg} \mathrm{cm}^{-2} \mathrm{~s}^{-1}$, about $24 \%$ of which is due to the blackbody component.

We also attempted to fit the soft part of the spectra with other emission models, such as mekal, thermal bremsstrahlung and broken power-law. In all these cases the results were worse than those obtained with the blackbody model, since the reduced chisquared was higher, the residuals were larger and/or the best-fit parameter values were unrealistic.

We did not find significant evidence for emission lines. By adding Gaussian components at various energies to the above model, we estimated upper limits of the order of $150 \mathrm{eV}$ for the equivalent width of lines in the 6-7 keV energy range.

\section{Phase-resolved spectroscopy}

Prompted by the results described above, we performed a phaseresolved spectroscopy in order to study in more detail the source behavior. We defined phase 0 by matching the folded light curve to that observed in 1996 with RossiXTE and extracted the background subtracted spectra for the same four phase intervals used

${ }^{1}$ Errors are at a $90 \%$ confidence level for a single interesting parameter.
Table 1. Best-fit spectral parameters for the phase-resolved spectroscopy of RX J0146.9+6121, in the case of the independent fit of the four spectra. Errors are at a $90 \%$ confidence level for a single interesting parameter. $N_{\mathrm{H}}$ and $k T_{\mathrm{BB}}$ are measured in units of $10^{21} \mathrm{~cm}^{-2}$ and $\mathrm{keV}$, respectively.

\begin{tabular}{c|cccc}
\hline \hline Spectral & \multicolumn{4}{|c}{ Phase Interval } \\
Parameter & $\mathrm{A}$ & $\mathrm{B}$ & $\mathrm{C}$ & $\mathrm{D}$ \\
\hline$N_{\mathrm{H}}$ & $4.8_{-0.7}^{+0.4}$ & $5.4_{-0.3}^{+0.4}$ & $5.0_{-0.7}^{+0.8}$ & $3.6_{-0.5}^{+0.6}$ \\
$\Gamma$ & $1.47_{-0.20}^{+0.19}$ & $1.39_{-0.08}^{+0.09}$ & $1.31_{-0.23}^{+0.35}$ & $1.06_{-0.21}^{+0.11}$ \\
$k T_{\mathrm{BB}}$ & $0.98_{-0.12}^{+0.08}$ & $1.05_{-0.08}^{+0.10}$ & $1.34_{-0.18}^{+0.17}$ & $1.21_{-0.16}^{+0.21}$ \\
\hline$f_{\mathrm{TOT}}^{a}$ & 1.81 & 3.22 & 1.74 & 1.00 \\
$f_{\mathrm{PL}}^{a}$ & 1.34 & 2.52 & 1.10 & 0.70 \\
& $(74 \%)$ & $(78 \%)$ & $(63 \%)$ & $(70 \%)$ \\
$f_{\mathrm{BB}}^{a}$ & 0.47 & 0.70 & 0.64 & 0.30 \\
& $(26 \%)$ & $(22 \%)$ & $(37 \%)$ & $(30 \%)$ \\
\hline$\chi_{v}^{2} /$ d.o.f. & $0.972 / 308$ & $1.021 / 992$ & $1.085 / 352$ & $1.131 / 328$ \\
\hline
\end{tabular}

a Unabsorbed flux in the energy range $0.3-10 \mathrm{keV}$, in units of $10^{-11} \mathrm{erg} \mathrm{cm}^{-2} \mathrm{~s}^{-1}$.

Table 2. Best-fit values for the black-body and power-law normalizations, when the four spectra are fitted simultaneously with common values of $N_{\mathrm{H}}\left((5.8 \pm 0.1) \times 10^{21} \mathrm{~cm}^{-2}\right), \Gamma(1.60 \pm 0.02)$ and $k T_{\mathrm{BB}}$ $\left(1.36_{-0.03}^{+0.04} \mathrm{keV}\right)$. Errors are at a $90 \%$ confidence level for a single interesting parameter.

\begin{tabular}{c|cccc}
\hline \hline Spectral & \multicolumn{4}{|c}{ Phase Interval } \\
Parameter & $\mathrm{A}$ & $\mathrm{B}$ & $\mathrm{C}$ & $\mathrm{D}$ \\
\hline$R_{\mathrm{BB}}^{a}$ & $76 \pm 3$ & $130 \pm 2$ & $121 \pm 2$ & $87 \pm 2$ \\
$I_{\mathrm{PL}}^{b}$ & $2.24_{-0.03}^{+0.07}$ & $3.25_{-0.03}^{+0.05}$ & $1.32_{-0.06}^{+0.03}$ & $0.89_{-0.03}^{+0.01}$ \\
$f_{\mathrm{TOT}}^{c}$ & 2.04 & 3.42 & 1.77 & 1.06 \\
$f_{\mathrm{PL}}^{c}$ & 1.69 & 2.46 & 1.00 & 0.67 \\
& $(83 \%)$ & $(72 \%)$ & $(56 \%)$ & $(63 \%)$ \\
$f_{\mathrm{BB}}^{c}$ & 0.35 & 0.96 & 0.77 & 0.39 \\
& $(17 \%)$ & $(28 \%)$ & $(44 \%)$ & $(37 \%)$ \\
\hline
\end{tabular}

${ }^{a}$ Radius of the blackbody component (in metres) for a source distance of $2.5 \mathrm{kpc}$.

$b$ Intensity of the power-law component in units of $10^{-3} \mathrm{ph} \mathrm{cm}^{-2} \mathrm{~s}^{-1} \mathrm{keV}^{-1}$ at $1 \mathrm{keV}$.

$c$ Unabsorbed flux in the energy range $0.3-10 \mathrm{keV}$, in units of $10^{-11} \mathrm{erg} \mathrm{cm}^{-2} \mathrm{~s}^{-1}$.

in Mereghetti et al. (2000) and indicated in Fig. 2. The first step was to fit all of them with the best-fit power law plus blackbody model of the phase averaged spectrum, leaving only the relative normalization factors free to vary. The ratios of the four spectra of each instrument to these renormalized average models show significant residuals and clearly demonstrate the spectral variability as a function of the pulse phase.

We then fitted the four spectra independently. In all cases, the absorbed power-law model was not satisfactory, while the addition of a black-body component significantly improved the fit. Therefore we used this model for all the spectra, leaving all the parameters free to vary: the results are reported in Table 1.

In order to investigate the relative variations of the two components with the period phase, we also simultaneously fitted the four spectra forcing common values for $N_{\mathrm{H}}, \Gamma$ and $k T_{\mathrm{BB}}$. In this case we obtained $N_{\mathrm{H}}=(5.8 \pm 0.1) \times 10^{21} \mathrm{~cm}^{-2}, \Gamma_{\mathrm{PL}}=1.60 \pm 0.02$ and $k T_{\mathrm{BB}}=1.36_{-0.03}^{+0.04} \mathrm{keV}$, with $\chi_{v}^{2} /$ d.o.f. $=1.117 / 1989$; the corresponding normalization values are reported in Table 2 . In this interpretation the spectral changes as a function of the phase are reproduced by the variations in the relative contribution of the two components. 
Table 3. Best-fit values for the power-law parameters, when the four spectra are fitted simultaneously with common values of $N_{\mathrm{H}}\left(\left(6.1_{-0.1}^{+0.3}\right) \times\right.$ $\left.10^{21} \mathrm{~cm}^{-2}\right), k T_{\mathrm{BB}}\left(1.40_{-0.04}^{+0.02} \mathrm{keV}\right)$ and $R_{\mathrm{BB}}\left(111_{-4}^{+1} \mathrm{~m}\right)$. Errors are at $90 \%$ confidence level for a single interesting parameter.

\begin{tabular}{c|cccc}
\hline \hline Spectral & \multicolumn{4}{|c}{ Phase Interval } \\
Parameter & $\mathrm{A}$ & $\mathrm{B}$ & $\mathrm{C}$ & $\mathrm{D}$ \\
\hline$\Gamma$ & $1.98_{-0.04}^{+0.03}$ & $1.57_{-0.02}^{+0.02}$ & $1.56_{-0.03}^{+0.04}$ & $2.63_{-0.08}^{+0.13}$ \\
$I_{\mathrm{PL}}^{a}$ & $2.40_{-0.06}^{+0.06}$ & $3.47_{-0.04}^{+0.05}$ & $1.39_{-0.05}^{+0.03}$ & $0.97_{-0.05}^{+0.04}$ \\
$f_{\mathrm{TOT}}^{b}$ & 2.08 & 3.43 & 1.81 & 1.19 \\
$f_{\mathrm{PL}}^{b}$ & 1.36 & 2.71 & 1.09 & 0.47 \\
& $(65 \%)$ & $(79 \%)$ & $(60 \%)$ & $(40 \%)$ \\
$f_{\mathrm{BB}}^{b}$ & 0.72 & 0.72 & 0.72 & 0.72 \\
& $(35 \%)$ & $(21 \%)$ & $(40 \%)$ & $(60 \%)$ \\
\hline
\end{tabular}

a Intensity of the power-law component in units of $10^{-3} \mathrm{ph} \mathrm{cm}^{-2} \mathrm{~s}^{-1} \mathrm{keV}^{-1}$ at $1 \mathrm{keV}$.

${ }^{b}$ Unabsorbed flux in the energy range $0.3-10 \mathrm{keV}$, in units of $10^{-11} \mathrm{erg} \mathrm{cm}^{-2} \mathrm{~s}^{-1}$.

However, the above results do not necessarily imply that the soft component is pulsed. In fact an acceptable fit $\left(\chi_{v}^{2} /\right.$ d.o.f. $=$ $1.097 / 1989$ ) can also be obtained by imposing that the blackbody component parameters be the same in the four spectra, as shown in Table 3.

The above results are summarized in Fig. 5, where we show the pulse-phase dependence of the black-body temperature, the power-law photon index and the unabsorbed flux of the two components for both fits. Since they have a similar statistical quality, we can neither confirm nor deny that the thermal component is variable.

\section{Discussion}

The XMM-Newton data described here were obtained almost 6 years after the latest X-ray observation of RX J0146.9+6121, which was performed by BeppoSAX on 1998 February 3 (Mereghetti et al. 2000). Therefore it is interesting to compare our results with those obtained in the past. In Fig. 6 we show the long term evolution of the source spin period and luminosity since the time of the EXOSAT discovery in 1984. If we exclude the first observation, when the source was in outburst, a linear fit of all the periods gives a spin-up at an average rate of $\dot{P}=-\left(4.6_{-0.2}^{+0.1}\right) \times 10^{-8} \mathrm{~s} \mathrm{~s}^{-1}$, similar to that measured until 1998. This result suggests that during the 6 years between the BeppoSAX and the XMM-Newton observations, the momentum transfer onto the neutron star has proceeded with no major changes. The flux detected by XMM-Newton corresponds to a luminosity of $\sim 1.5 \times 10^{34} \mathrm{erg} \mathrm{s}^{-1}$, a factor $\sim 2$ lower than the minimum level observed in the previous years, indicating that, after the outburst of July 1997 (Haberl et al. 1998), RX J0146.9+6121 has been continuously fading.

Comparison with the RossiXTE results reported in Fig. 2 of Mereghetti et al. (2000) shows that, despite the luminosity variation, the shape of the pulse profiles in the energy interval common to the two instruments $(2-10 \mathrm{keV})$ has not changed. The XMM-Newton data also confirm that, above $2 \mathrm{keV}$, there is a significant spectral softening in the initial rising part of the pulse (phase interval A).

The main advantage of the data reported here, with respect to previous observations of this source, is the better coverage of the energy range below $2 \mathrm{keV}$. In comparison to the BeppoSAX results, the phase averaged spectroscopy gives a smaller hydrogen column density: $N_{\mathrm{H}}=\left(5.09_{-0.23}^{+0.24}\right) \times 10^{21} \mathrm{~cm}^{-2}$ instead of
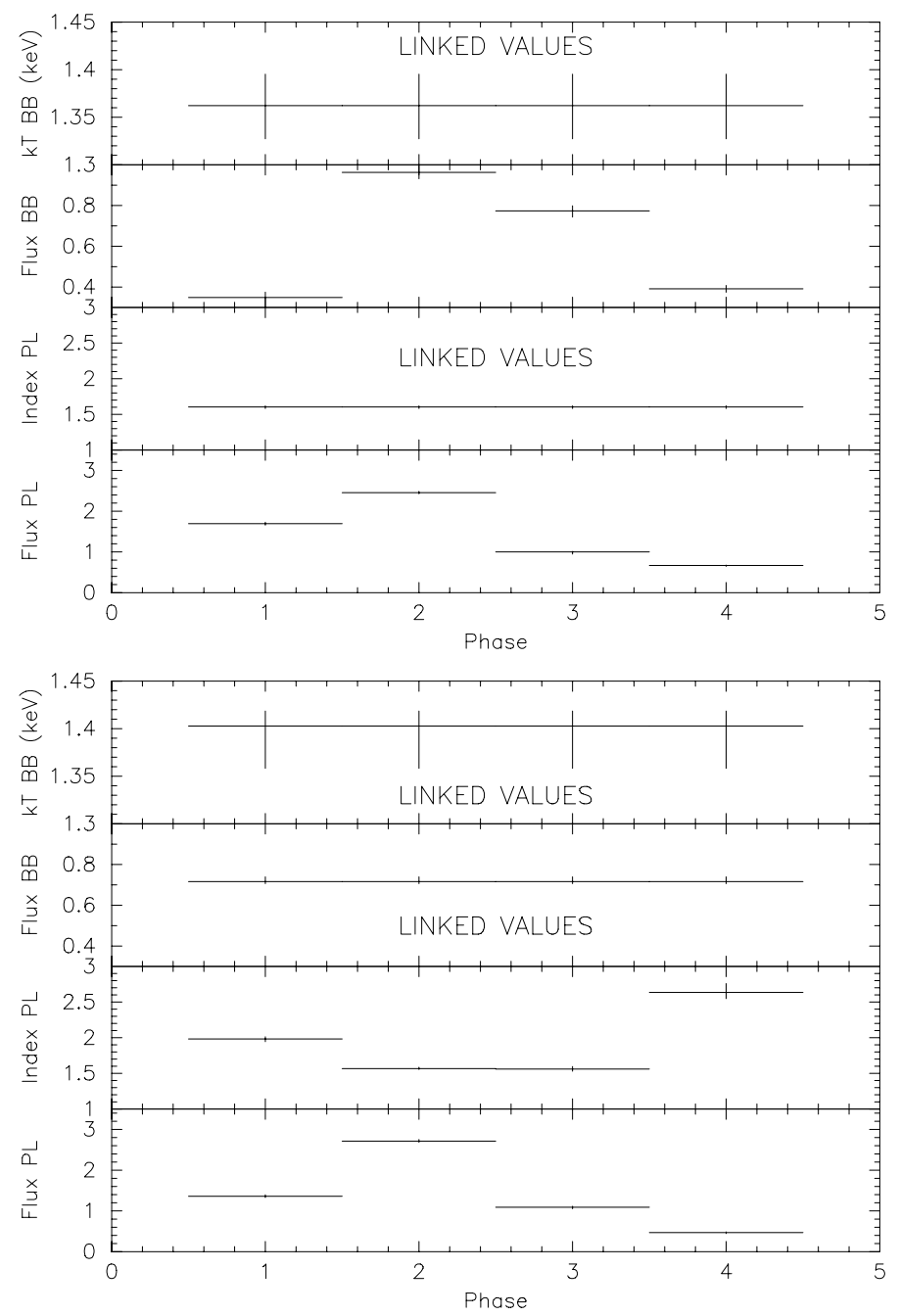

Fig. 5. Pulse-phase dependence of the black-body temperature, the power-law photon index and the unabsorbed flux of the two components (in units of $10^{-11} \mathrm{erg} \mathrm{cm}^{-2} \mathrm{~s}^{-1}$ ), in the case of a common temperature and index (top) and of a common black-body (bottom).

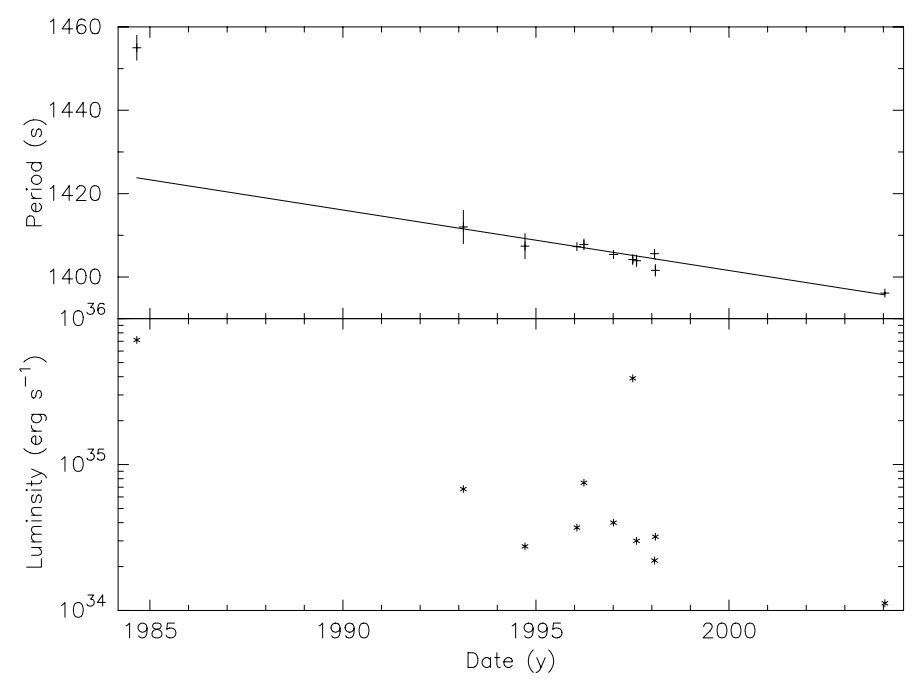

Fig. 6. Pulse period and luminosity history of RX J0146.9+6121 from 1984 August 27 to 2004 January 15. The solid line in the upper panel is the best-fit linear model of the reported values and has a slope $\dot{P}=$ $-4.6 \times 10^{-8} \mathrm{~s} \mathrm{~s}^{-1}$. The luminosities of the lower panel are based on the unabsorbed flux in the $2-10 \mathrm{keV}$ energy range and on a source distance of $2.5 \mathrm{kpc}$. 
$(1.2 \pm 0.3) \times 10^{22} \mathrm{~cm}^{-2}$. This value is in better agreement with that expected from the optical observations which give $E(B-V)=$ 0.93 (Reig et al. 1997). Assuming $A_{\mathrm{V}}=3.1 E(B-V)$ and the average relation $A_{\mathrm{V}}=N_{\mathrm{H}} \times 5.59 \times 10^{-22} \mathrm{~cm}^{-2}$ between optical extinction and X-ray absorption (Predehl \& Schmitt 1995), this would predict $N_{\mathrm{H}}=5.16 \times 10^{21} \mathrm{~cm}^{-2}$.

Moreover, we find evidence of a thermal component that was never observed before in this source. In recent years, similar soft excesses have been detected in several high mass X-ray binary pulsars. The properties of these sources are summarized in Table 4. Hickox et al. (2004) showed that a soft excess has been detected in all the X-ray pulsars with a sufficiently high flux and small absorption. In fact, most of the soft excess sources are at small distances and/or away from the Galactic plane (most of them are in the Magellanic Clouds). This suggests that the presence of a soft spectral component could be a very common, if not an ubiquitous, feature intrinsic to X-ray pulsars. These authors concluded that the origin of the soft component is related to the source total luminosity. When $L_{\mathrm{X}} \geq 10^{38} \mathrm{erg} \mathrm{s}^{-1}$ the luminosity and the shape of the soft component can be explained only by reprocessing of hard X-rays from the neutron star by optically thick accreting material, most likely near the inner edge of the accretion disk. In less luminous sources, with $L_{\mathrm{X}} \leq 10^{36} \mathrm{erg} \mathrm{s}^{-1}$, the soft excess can be due to other processes, such as emission by photo-ionized or collisionally heated diffuse gas or thermal emission from the surface of the neutron star. Finally, in the sources of intermediate luminosity, either or both of these types of emission can be present.

In Fig. 7 we have plotted the X-ray luminosity versus interstellar absorption of the pulsars with a soft excess. We can see that RX J0146.9+6121, together with X Persei and $3 \mathrm{~A} 0535+262$, is at least one order of magnitude less luminous than all the other pulsars displaying a soft excess. We note that the ratio between the unabsorbed fluxes of the thermal and the power-law components in RX J0146.9+6121 agrees well with the average value measured in the other sources. However, unlike some of the other sources, the soft excess could be fitted only with a black-body component, while any other emission model was rejected.

Based on the results obtained by Hickox et al. (2004), the luminosity of RX J0146.9+6121 is too small for an interpretation of the soft excess in terms of reprocessing of the hard $\mathrm{X}$-ray photons in optically thick accreting material. Moreover, both the thermal emission and the hard X-ray reprocessing in a diffuse, optically thin gas around the neutron star are unlikely, since these processes would not give a black-body spectrum. We therefore favor the interpretation of the soft excess in RX J0146.9+6121 as thermal emission from the neutron star polar cap. If we assume that the source is in the "accretor" status, with matter accretion on the NS surface, the blackbody emitting radius of $\sim 140 \mathrm{~m}$ is consistent with the expected size of the polar cap. In fact, if $M_{\mathrm{NS}}=1.4 M_{\odot}$ and $R_{\mathrm{NS}}=10^{6} \mathrm{~cm}$, the source luminosity of $\sim 10^{34} \mathrm{erg} \mathrm{s}^{-1}$ implies an accretion rate $\dot{M} \sim 5 \times 10^{13} \mathrm{~g} \mathrm{~s}^{-1}$ and, adopting $B_{\mathrm{NS}}=10^{12} \mathrm{G}$, a magnetospheric radius $R_{\mathrm{m}} \simeq 2.4 \times 10^{9} \mathrm{~cm}$ (Campana et al. 1998). In this case, based on the relation $R_{\mathrm{col}} \sim R_{\mathrm{NS}}\left(R_{\mathrm{NS}} / R_{\mathrm{m}}\right)^{0.5}$ (Hickox et al. 2004), we would obtain $R_{\text {col }} \sim 200 \mathrm{~m}$. If this description is correct, we would expect to observe some variability of the soft component along the pulse phase. From this point of view the phase-resolved spectroscopy provides no conclusive results, since it proves that both a variable and a constant thermal component can account for the observed spectral variability.

RX J0146.9+6121 is in many respects very similar to X Per and $3 \mathrm{~A} 0535+262$, which are very low luminosity Be/NS binary

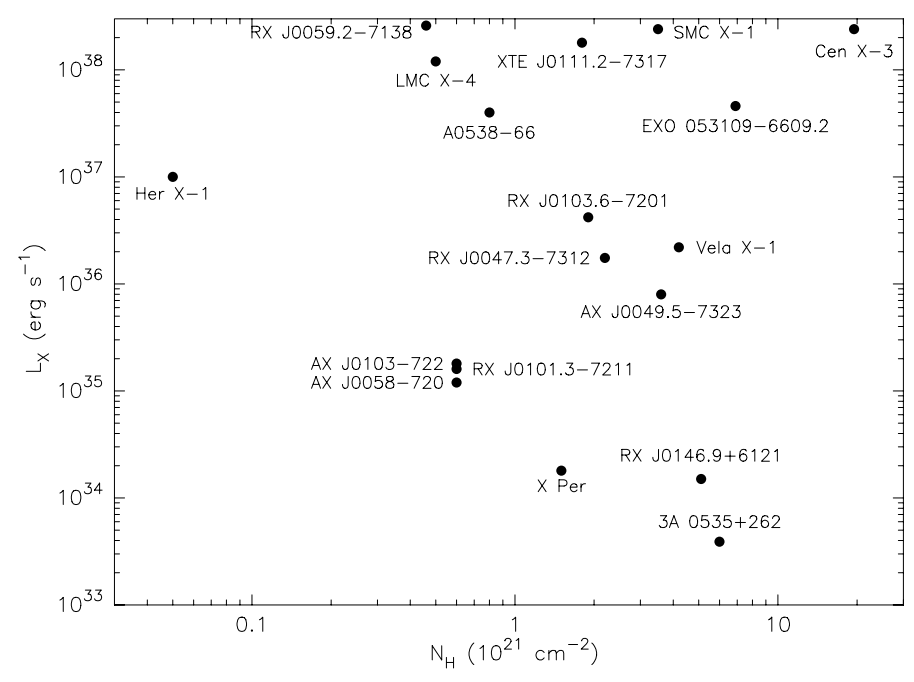

Fig. 7. Total X-ray luminosity of the sources of Table 4 as a function of the interstellar absorption.

with a long pulse period. The soft excesses of these sources have similar properties, since they tend to have a higher temperature $(>1 \mathrm{keV})$ and a smaller emission radius $(\sim 0.1 \mathrm{~km})$ compared to the soft excesses observed in high luminosity systems, which have a temperature of about $0.1 \mathrm{keV}$ and an emission radius of a few hundred $\mathrm{km}$. In these three low luminosity systems the soft excess contributes 25-35\% of the total flux (Coburn et al. 2001; Mukherjee \& Paul 2005). Also in the case of X Per and 3 A $0535+262$ this excess has been attributed to the emission from the polar caps.

\section{Conclusions}

We have reported the analysis of the data collected by XMM-Newton in a $\sim 42 \mathrm{ks}$ observation of the Be/neutron-star X-ray pulsar RX J0146.9+6121.

The unabsorbed flux corresponds to a source luminosity $L_{\mathrm{X}} \sim 1 \times 10^{34} \mathrm{erg} \mathrm{s}^{-1}$ in the $2-10 \mathrm{keV}$ energy range, about $50 \%$ lower than the lowest level ever observed from this source, indicating a monotonic source fading over long time scales.

Thanks to the high effective area of XMM-Newton also at low energies, we could perform the first accurate spectral study below $2 \mathrm{keV}$ for this source. In the phase-averaged spectrum we have revealed the presence of a significant soft excess over the primary power-law component: this excess can be described by a black-body with $k T_{\mathrm{BB}} \sim 1 \mathrm{keV}$, while any attempt to fit it with a different emission model was unsuccessful.

The phase-resolved spectroscopy has confirmed the large spectral variability along the pulse period already observed above $2 \mathrm{keV}$. Unfortunately, with the current data, it is not possible to derive compelling results on the phase variability of the soft excess component. Although the emission below $2 \mathrm{keV}$ is clearly pulsed and the low energy part of the spectrum varies with the phase, we have shown that such variations can be explained equally well by changes in the blackbody component or in the power law component alone.

Clearly the relatively small distance and low interstellar absorption toward RX J0146.9+6121 plays a role in the possibility of detecting a soft excess in such a low luminosity pulsar. Comparison with other X-ray binary pulsars shows that, so far, a soft excess has been detected only in much brighter sources. The data reported here support the hypothesis that a soft 


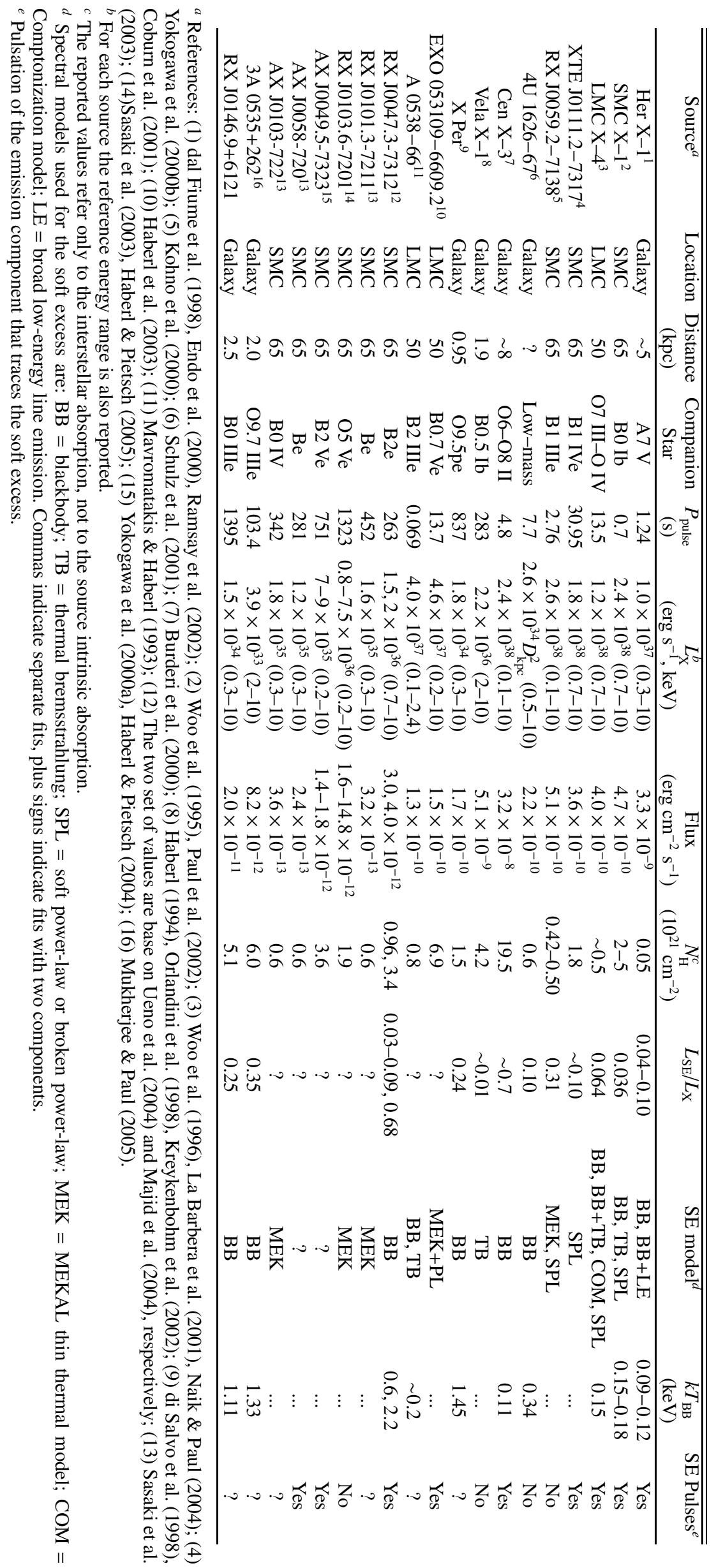


thermal component is a ubiquitous emission feature of this class of sources. In this sense, it would be very interesting to use the large collecting area of XMM-Newton in long observations of the faintest and longest period Be binaries, both in the SMC and in the Milky Way, such as, for example, the persistent low luminosity systems RX J0440.9+4431 and RX J1037.5-5647 (Reig \& Roche 1999).

Acknowledgements. We thank A. Tiengo for his useful suggestions. The $X M M-N e w t o n$ data analysis is supported by the Italian Space Agency (ASI), through contract ASI/INAF I/023/05/0.

\section{References}

Burderi, L., Di Salvo, T., Robba, N. R., La Barbera, A., \& Guainazzi, M. 2000, ApJ, 530, 429

Campana, S., Colpi, M., Mereghetti, S., Stella, L., \& Tavani, M. 1998, A\&AR, 8, 279

Coburn, W., Heindl, W. A., Gruber, D. E., et al. 2001, ApJ, 552, 738

Coe, M. J., Everall, C., Norton, A. J., et al. 1993, MNRAS, 261, 599

dal Fiume, D., Orlandini, M., Cusumano, G., et al. 1998, A\&A, 329, L41

di Salvo, T., Burderi, L., Robba, N. R., \& Guainazzi, M. 1998, ApJ, 509, 897

Endo, T., Nagase, F., \& Mihara, T. 2000, PASJ, 52, 223

Fabregat, J., Torrejon, J. M., Reig, P., et al. 1996, A\&AS, 119, 271

Haberl, F. 1994, A\&A, 288, 791

Haberl, F., \& Sasaki, M. 2000, A\&A, 359, 573

Haberl, F., \& Pietsch, W. 2004, A\&A, 414, 667

Haberl, F., \& Pietsch, W. 2005, A\&A, 438, 211

Haberl, F., Angelini, L., \& Motch, C. 1998, A\&A, 335, 587

Haberl, F., Dennerl, K., \& Pietsch, W. 2003, A\&A, 406, 471

Hellier, C. 1994, MNRAS, 271, L21

Hickox, R. C., Narayan, R., \& Kallman, T. R. 2004, ApJ, 614, 881

Israel, G. L., Campana, S., Covino, S., et al. 2000, ApJ, 531, L131

Kharchenko, N. V., Piskunov, A. E., Röser, S., Schilbach, E., \& Scholz, R.-D. 2005, A\&A, 438, 1163
Kohno, M., Yokogawa, J., \& Koyama, K. 2000, PASJ, 52, 299

Kreykenbohm, I., Coburn, W., Wilms, J., et al. 2002, A\&A, 395, 129

La Barbera, A., Burderi, L., Di Salvo, T., Iaria, R., \& Robba, N. R. 2001, ApJ, 553,375

Leahy, D. A. 1987, A\&A, 180, 275

Macomb, D. J., Fox, D. W., Lamb, R. C., \& Prince, T. A. 2003, ApJ, 584, L79

Majid, W. A., Lamb, R. C., \& Macomb, D. J. 2004, ApJ, 609, 133

Mavromatakis, F., \& Haberl, F. 1993, A\&A, 274, 304

Mereghetti, S., Stella, L., \& de Nile, F. 1993, A\&A, 278, L23

Mereghetti, S., Tiengo, A., Israel, G. L., \& Stella, L. 2000, A\&A, 354, 567

Mukherjee, U., \& Paul, B. 2005, A\&A, 431, 667

Nagase, F. 2002, J. Astrophys. Astron., 23, 59

Naik, S., \& Paul, B. 2004, ApJ, 600, 351

Orlandini, M., dal Fiume, D., Frontera, F., et al. 1998, A\&A, 332, 121

Pandey, A. K., Upadhyay, K., Ogura, K., et al. 2005, MNRAS, 358, 1290

Paul, B., Nagase, F., Endo, T., et al. 2002, ApJ, 579, 411

Pigulski, A., Kopacki, G., \& Kołaczkowski, Z. 2001, A\&A, 376, 144

Predehl, P., \& Schmitt, J. H. M. M. 1995, A\&A, 293, 889

Ramsay, G., Zane, S., Jimenez-Garate, M. A., den Herder, J.-W., \& Hailey, C. J. 2002, MNRAS, 337, 1185

Reig, P., Fabregat, J., Coe, M. J., et al. 1997, A\&A, 322, 183

Reig, P., \& Roche, P. 1999, MNRAS, 306, 100

Sasaki, M., Pietsch, W., \& Haberl, F. 2003, A\&A, 403, 901

Schulz, N. S., Chakrabarty, D., Marshall, H. L., et al. 2001, ApJ, 563, 941

Strüder, L., Briel, U., Dennerl, K., et al. 2001, A\&A, 365, L18

Tapia, M., Costero, R., Echevarria, J., \& Roth, M. 1991, MNRAS, 253, 649

Turner, M. J. L., Abbey, A., Arnaud, M., et al. 2001, A\&A, 365, L27

Ueno, M., Yamaguchi, H., Takagi, S.-I., Yokogawa, J., \& Koyama, K. 2004, PASJ, 56, 175

White, N. E., Mason, K. O., Giommi, P., et al. 1987, MNRAS, 226, 645

Woo, J. W., Clark, G. W., Blondin, J. M., Kallman, T. R., \& Nagase, F. 1995, ApJ, 445, 896

Woo, J. W., Clark, G. W., Levine, A. M., Corbet, R. H. D., \& Nagase, F. 1996, ApJ, 467, 811

Yokogawa, J., Imanishi, K., Tsujimoto, M., Koyama, K., \& Nishiuchi, M. 2003, PASJ, 55, 161

Yokogawa, J., Imanishi, K., Ueno, M., \& Koyama, K. 2000a, PASJ, 52, L73

Yokogawa, J., Paul, B., Ozaki, M., et al. 2000b, ApJ, 539, 191 